Document downloaded from:

http://hdl.handle.net/10251/47700

This paper must be cited as:

Luna, R.; Millan, C.; Domingo, M.; et ál..(2012). Upgraded sublimation energy determination procedure for icy films. Vacuum. 86(12):1969-1973. doi:10.1016/j.vacuum.2012.05.010.

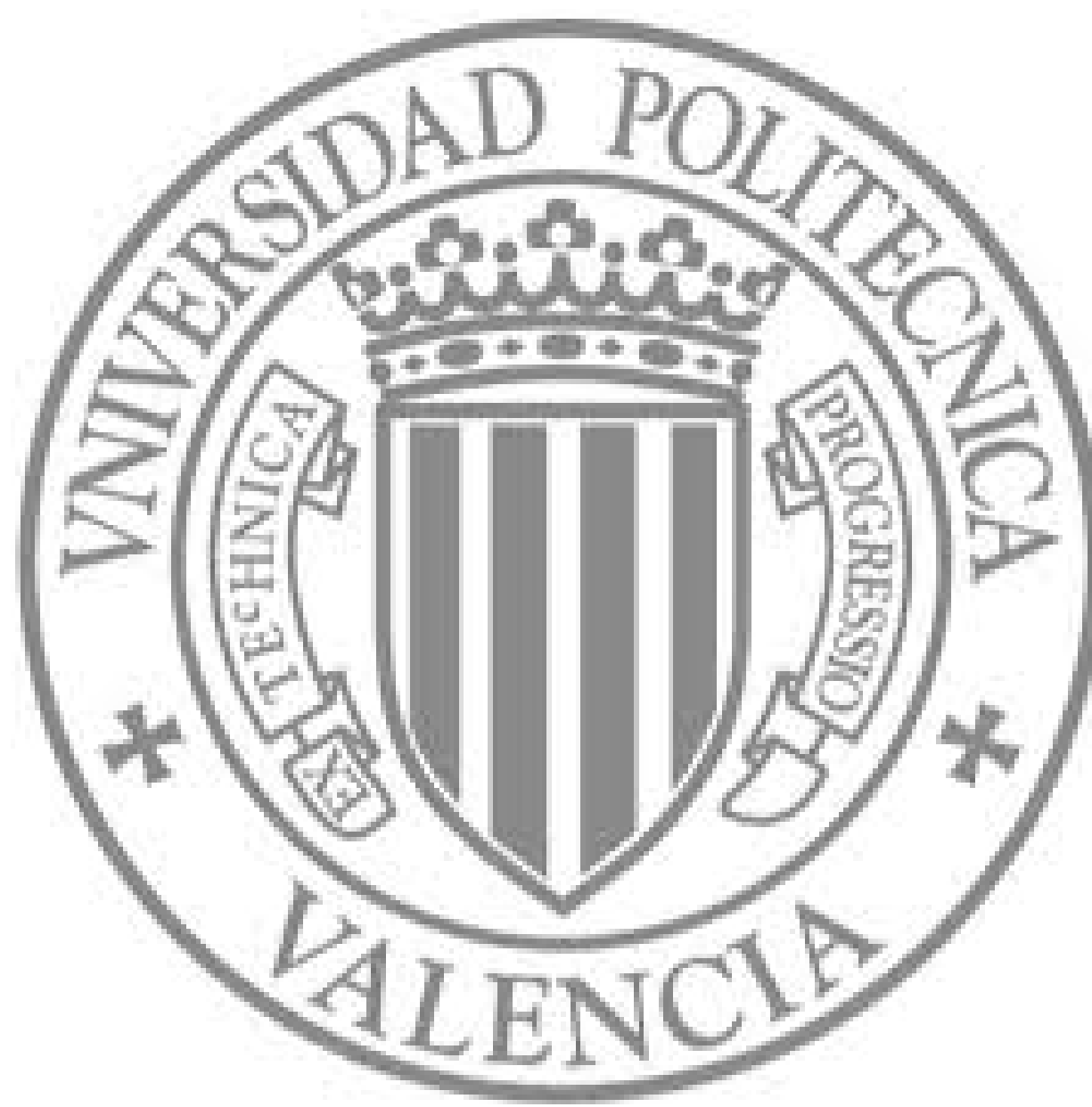

The final publication is available at

http://dx.doi.org/10.1016/j.vacuum.2012.05.010

Copyright Elsevier 


\title{
Upgraded sublimation energy determination procedure for icy films
}

\author{
R. Luna ${ }^{a}$, C. Millán ${ }^{\text {a }}$, M. Domingo ${ }^{\text {a }}$, C. Santonja ${ }^{a}$, M. Satorre $^{\text {a }}$ \\ ${ }^{a}$ Centro de Tecnologías Físicas. Universitat Politècnica de València, 46022 València, Spain
}

\begin{abstract}
A method to determine the sublimation energy of a bulk ice in high vacuum systems, allowing other simultaneous analysis techniques, is presented. Variation in frequency of a quartz crystal microbalance, due to sublimating material, during a zeroth-order desorption consents to obtain this energy. $\mathrm{CO}_{2}$ sublimation energy is obtained to check this method, its value is coherent with that reported in the literature. Our method permits to simplify the setup used so far by other authors, and to obtain relevant parameters for ices simultaneously. The procedure explained here corrects the temperature frequency dependance of the microbalance and the effect of contaminants by using an unique microbalance.
\end{abstract}

Key words: ice films, sublimation energy, high vacuum and low temperature laboratory, quartz crystal microbalance

\section{Introduction}

The energy of sublimation $E_{s u b}$ is defined as the heat required to sublime one mole of a substance $[1,2]$. This parameter is relevant to compute several thermodynamical magnitudes such as the reticular energy of ionic compounds, the energy of formation in gas phase from the energy of formation in condensed phase, or the sublimation rate of molecules from their bulk ice.

The energy of sublimation can be determined directly or indirectly. Santos et al. [3] determine this energy directly by means of calorimetry during the phase transition. Indirect methods measure a parameter related to the sublimation energy. In many cases it is determined from the vapor pressure of the substance under study at different temperatures and then, using the Clapeyron equation, the energy of sublimation is derived [4]. Vapor pressure is usually calculated by measuring the mass flow from an oven at controlled temperature by using a Knudsen effu- sive cell [5]. There are, among others, several ways to determine this mass flow: a quartz crystal microbalance (QCMB) [6,7]; a torsion pendulum [8]; a fluorescence spectrometer [9]; etc.

The use of a Knudsen cell hampers to study directly the solid phase with other analysis techniques to obtain simultaneously additional parameters. Characterization of an ice needs several parameters as: density, porosity, refractive index, etc. Therefore, simultaneity is desirable because it would guarantee results corresponding exactly to the same ice structure, another benefit of it is to economize on resources and time.

Bryson et al. [10] and Sack and Baragiola [11] used one QCMB, acting as deposition surface and detector simultaneously, to determine the vapor pressure of an ice [10]. Obtaining mass flow by means a microbalance simplifies the experimental setup clearing the surface of the ice. This setup provides enough free space to implement additional techniques simultaneously. In fact, Teolis et al. [12] have used the 
QCMB to calculate the $\mathrm{O}_{3}$ sublimation energy from the desorption rate and additionally they have studied the sample with infrared and UV-visible spectroscopy at the same time.

To determine the sublimation rate using a QCMB it is necessary to consider two undesired effects: the frequency temperature dependance of the microbalance, and the deposition of contaminants on it.

Teolis et al. [12] mounted another QCMB on the back of the target, which was not exposed to gases, to eliminate the effect of temperature. However, using an additional QCMB as a reference, requires that both balances are temperature synchronized, and their behaviour against temperature is the same. So it is crucial to assure the same thermal contact in the setup, and to know the exact response for both QCMB.

Concerning the second undesired effect, these authors use ultra high vacuum (UHV) systems, in which the presence of contaminants is negligible (contaminants $<0.1 \%$ of total mass deposited). But this problem was still unsolved for high vacuum (HV) systems where the presence of contaminants is 2 or 3 orders of magnitude higher.

The method presented below consents to use HV systems to determine the energy of sublimation simultaneously with other analysis techniques. This procedure takes the advantages of the QCMB, avoiding the complexity of using a second microbalance, because it is possible to identify its frequency behaviour versus temperature. Additionally, it is remarkable that it is not necessary to stop and restart warming up to obtain the sublimation energy, as the methods based on the vapor pressure do.

\section{Experimental setup}

The basic components of our experimental configuration (Fig. 1) are a high vacuum and low temperature system, a QCMB, two lasers and a quadrupole mass spectrometer (QMS) [14].

Vacuum in the chamber is obtained with a turbomolecular pump backed by a rotary pump. The first stage of a closed-cycle He cryostat (40 K) thermally connected to a shield protector acts as a cryopump providing a base pressure below $10^{-7}$ mbar measured with an ITR IoniVac transmiter (5\% in accuracy). The composition and the partial pressure of gases are monitored by means the QMS (AccuQuad RGA 100 with a resolution of $\sim 0.5 \mathrm{amu})$. The second stage of the cryostat (hereafter cold finger) con-

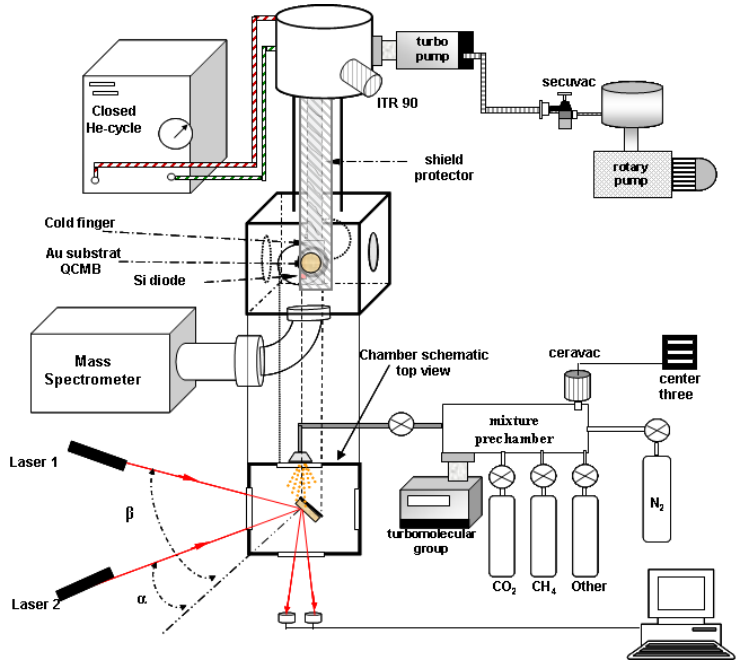

Fig. 1. Experimental setup.

sents to reach $10 \mathrm{~K}$ at the sample holder, in thermal contact with the QCMB (Q-Sense X 301, AT-cut, 0216/1, $5 \mathrm{MHz}$ ).

The temperature in the sample (QCMB) is operated by the Intelligent Temperature Controller ITC 503S (Oxford Instruments). It uses the feedback of a silicon diode sensor (Scientific Instruments) located just beside the quartz, that allows the temperature to vary between 10.0 and $300.0 \pm 0.1 \mathrm{~K}$, by means a resistive heater.

Gas under study is charged in a prechamber at a suitable pressure measured with a Ceravac CTR 90 (Leybold Vacuum) whose accuracy is $0.2 \%$, provided with a ceramic sensor not influenced by the gas type (range $10^{4}$, FS 100 torr).

Gas enters the chamber through a needle valve (Leybold D50968) which regulates the gas flow. During the ice growth on the QCMB, interferometric patterns are obtained by means of two He-Ne lasers $(632.8 \mathrm{~nm})$ located at two different angles $\alpha$ and $\beta$.

The following chemical has been used in this research: $\mathrm{CO}_{2}-99.998$ (Praxair). To preserve gas purity, the whole pre-chamber is evacuated with a turbomolecular group pumping speed $33 \mathrm{l} \mathrm{s}^{-1}$, ultimate pressure $<10^{-7}$ mbar $)$.

\section{Fundamentals}

\subsection{The quartz crystal microbalance}

The QCMB uses the piezoelectric qualities of a quartz crystal . An electric field applied to the gold coated crystal causes a shear deformation (parallel 


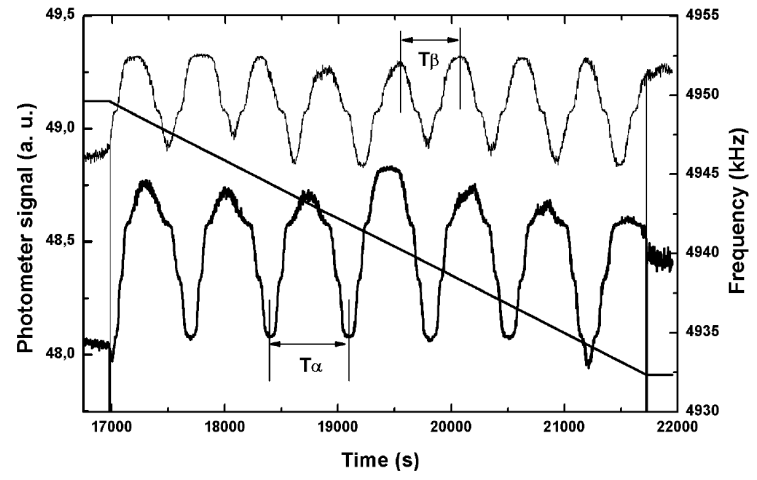

Fig. 2. Interferometric patterns at two angles of incidence, $\beta$ (upper curve) and $\alpha$ (lower curve), being $\beta>\alpha$ , and frequency variation versus time (straight line) during the growth of a $\mathrm{CO}_{2}$ ice film.

Table 1

Parameters obtained for the linear fit $(\mathrm{f}=\mathrm{A}+\mathrm{B} \cdot \mathrm{t}$ ) of frequency (f) versus time ( $\mathrm{t}$ ) for a $\mathrm{CO}_{2}$ deposition experiment of around 80 minutes.

\begin{tabular}{|c|c|}
\hline Parameter & Value \\
\hline $\mathrm{A}$ & $5011300 \pm 5 \mathrm{~Hz}$ \\
$\mathrm{~B}$ & $-3.6339 \pm 0.0003 \mathrm{~Hz} \mathrm{~s}^{-1}$ \\
\hline $\mathrm{r}$ & -0.99999 \\
\hline
\end{tabular}

to the gold surface). The crystal resonates if an alternating electric field is applied at a particular frequency $f_{0}[15]$, given by:

$f_{0}=\frac{\sqrt{\mu}}{2 \cdot d \cdot \sqrt{\rho}}$

Where $\mu, \rho$ and $d$ are the shear modulus, the density and the thickness of the quartz crystal respectively.

The use of a working golden electrode causes a reduction in frequency $f_{0}$. Additionally, a damping can be caused by the deposition of mass on its surface. Assuming a thin, uniform, rigidly attached mass, the Sauerbrey Equation [15] relates frequency reduction $\left(\Delta f_{0}\right)$ to mass loading $(\Delta m)$ through the expression:

$\Delta f_{0}=-S \cdot \Delta m$

In this equation, $S$ is the Sauerbrey constant that must be determined for every experimental assembly. In our case a value of $64.9 \mathrm{~Hz} \mu \mathrm{g}^{-1}$ is obtained using the microbalance to asses the density of ices as $\mathrm{CO}_{2}, \mathrm{~N}_{2}$ and $\mathrm{CH}_{4}$, and comparing them with those reported by Roux et al. [16]. Results differ less than $2 \%$.

To check that the Sauerbrey equation is fulfilled in our experiments, Fig. 2 shows the quartz crystal response during a $\mathrm{CO}_{2}$ deposition experiment and the two interferometric laser signals at different angles of incidence vs time. Interferometric patterns depend on the thickness of the sample, not on the time elapsed during deposition. The constancy in the laser periods $\left(\mathrm{T}_{\alpha}\right.$ and $\left.\mathrm{T}_{\beta}\right)$ confirms a constant rate of mass deposition. As a consequence, the straight line in the microbalance output, means that the ice film satisfies the constraints of the Sauerbrey equations (see linear fit parameters in Table 1).

\subsection{Zeroth-Order desorption}

In this work we consider sublimation as the desorption of multilayer films from a surface (as previously implicitly used Teolis et al. [12]), despite sublimation is a process implying an equilibrium state. Therefore the desorption kinetics is essentially independent of the coverage, i.e. as molecules sublimate from the interface ice-vacuum, more molecules underneath are exposed. Consequently, the number of molecules in this interface remains constant. Thus, sublimation can be modelled as a zeroth-order desorption process described by the Polanyi-Wigner Equation [13]:

$r_{s u b}=-k \cdot n^{0}$

Where $r_{\text {sub }}$ is the sublimation rate, $n$ is the number of molecules on the surface and $k$ (molecules $\mathrm{cm}^{-2} \mathrm{~s}^{-1}$ ), is calculated by using the Arrhenius expression:

$k=A \cdot \exp \left(-\frac{E_{s u b}}{R \cdot T}\right)$

Here, $A$ is the preexponential sublimation factor, $R$ is the constant of ideal gases, $E_{s u b}$ is the sublimation energy and $\mathrm{T}$ is the absolute temperature. Then:

$r_{s u b}=-A \cdot \exp \left(-\frac{E_{s u b}}{R \cdot T}\right) \cdot n^{0}$

Taking into account equations 5 and 2 and a constant rate of warming up, the expression of zerothorder desorption rate versus the QCMB frequency variation, is obtained:

$\Delta f_{0}=A^{\prime} \cdot \exp \left(-\frac{E_{s u b}}{R \cdot T}\right) \cdot n^{0}$

This kind of process follows a behavior characterized by an exponential increasing of the desorption rate with $\mathrm{T}$, and an abrupt drop when all molecules have desorbed. 


\section{Experimental procedure}

\subsection{Setting the starting conditions}

First of all, the temperature of accretion is selected depending on the molecule under study. In order to achieve this accretion temperature and to reduce as much as possible contamination, the procedure to cool down the QCMB is as follows: the cryostat is connected cooling down the system except the cold finger which is maintained over the sublimation temperature of undesired contaminants, by means the ITC 503S controller. When the pressure is around $5 \cdot 10^{-8} \mathrm{mbar}$, the ITC is reprogrammed to the temperature of deposition, which is achieved in few minutes.

Once the temperature is stabilized, the needle valve is opened. Molecules prepared in the prechamber replenish the chamber randomly and are deposited onto the QCMB (background deposition). Pressure in the chamber during deposition is, at least, three orders of magnitude higher than the base pressure leading to a negligible amount of contaminants. When there is enough thickness to consider that a zeroth-order desorption will occur and it can be measured by our system (around $0.1 \mu \mathrm{m}$ ), the needle valve is closed, leading to an instantaneous drop in pressure [14]. After five minutes, the sample is heated at $1.0 \mathrm{~K} \mathrm{~min}^{-1}$. During the whole process the pumping system is working continuously.

\subsection{Measurements and data treatment}

As mentioned previously, two possible interferences must be determined and removed: the presence of contaminants (mainly water) and the effect of temperature drift in the QCMB.

To better expose the procedure of determining the energy of sublimation it is used the particular case of $\mathrm{CO}_{2}$. Fig. 3 shows the frequency output from the $\mathrm{QCMB}$ as a function of the temperature. It is possible to distinguish four different processes. At the beginning of the experiment the temperature is reduced from 95 down to the deposition temperature, $42 \mathrm{~K}$ in this example. Frequency decreases linearly, see Fig. 3, upper dotted arrow 1. Then, the sudden drop in frequency (more than $1000 \mathrm{~Hz}$, dotted arrow 2 ) is due to the accretion of ice onto the balance surface, at $42 \mathrm{~K}$. Next, the sample is heated at 1.0 $\mathrm{K} \mathrm{min}^{-1}$, increasing the frequency versus temperature (dotted arrow 3 ). This linear behaviour is bro- ken when the release of the gas starts at about $80 \mathrm{~K}$ up to close to $92 \mathrm{~K}$ (dotted arrow 4).
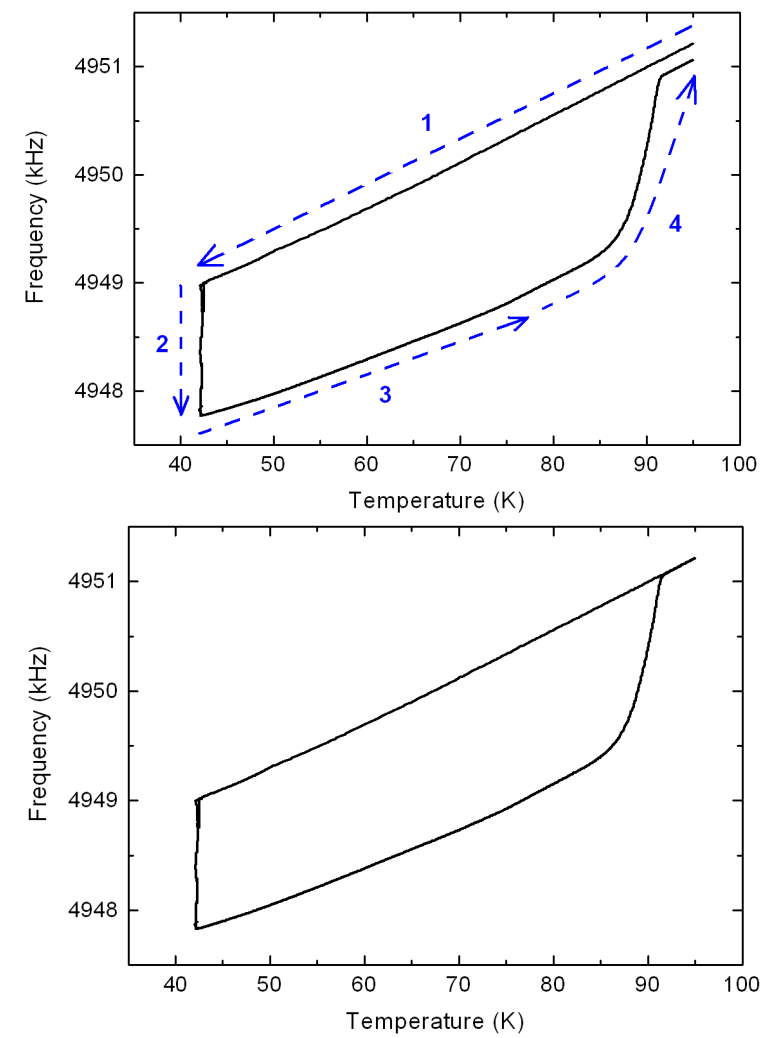

Fig. 3. QCMB response versus temperature. Top panel: before contaminants removal. Arrow 1: cool down; arrow 2: deposition at $42 \mathrm{~K}$; arrow 3 : warming up at $1 \mathrm{~K} \mathrm{~min}^{-1}$; arrow 4: sublimation. Bottom panel: QCMB response after contaminants removal.

Firstly, we describe how to remove the effect of the contaminants. Above $90 \mathrm{~K}$ (see around $92 \mathrm{~K}$ in Fig. 3, top panel, end of dotted arrow 4), when the desorption process is over, there exists a gap in frequency with respect to the initial value at the same temperature during the cooling process (beginning of dotted arrow 1). This shift in frequency quantifies the amount of contamination. Assuming a constant rate of contamination (since its pressure remains constant during the whole experiment) it is possible to subtract it, along the experiment, to use Equation 5 properly. In HV conditions the only relevant contaminant at temperature higher than $90 \mathrm{~K}$ is water $\left(T_{\text {sub }} \sim 180 \mathrm{~K}\right)$. Fig. 3, bottom panel shows the result of the QCMB frequency after weighted substraction of contaminants.

Secondly, the temperature influence on the balance signal is corrected. A linear relationship between frequency and temperature with a regression 

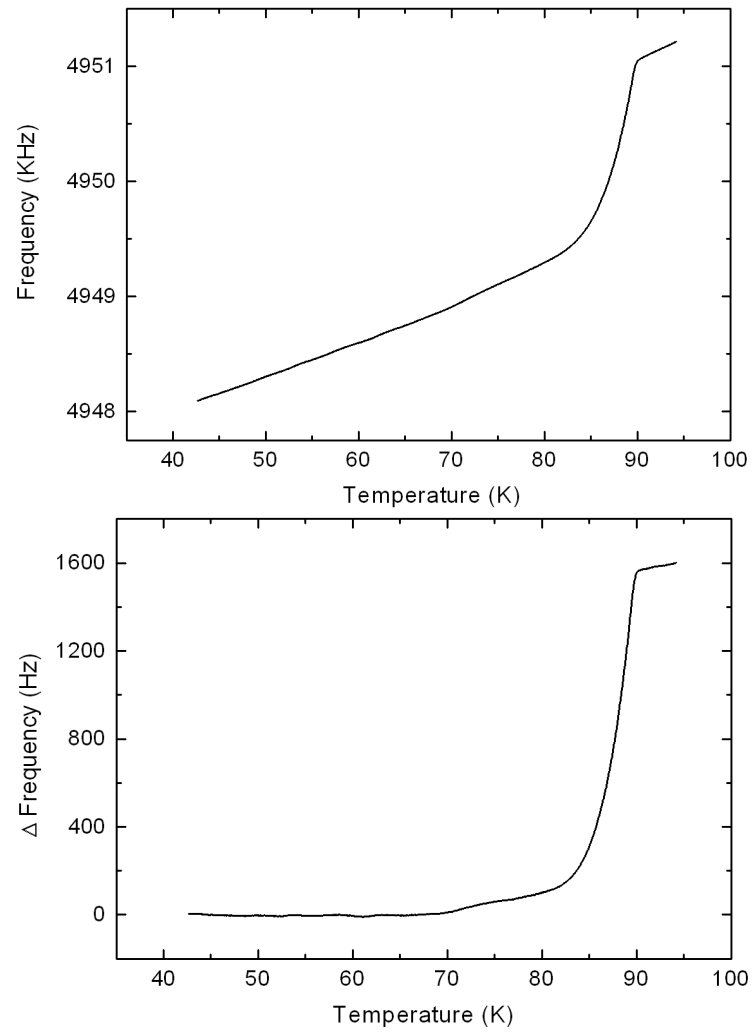

Fig. 4. Frequency as a function of temperature for $\mathrm{CO}_{2}$. Top panel: experimental data. Bottom panel: Temperature drift corrected.

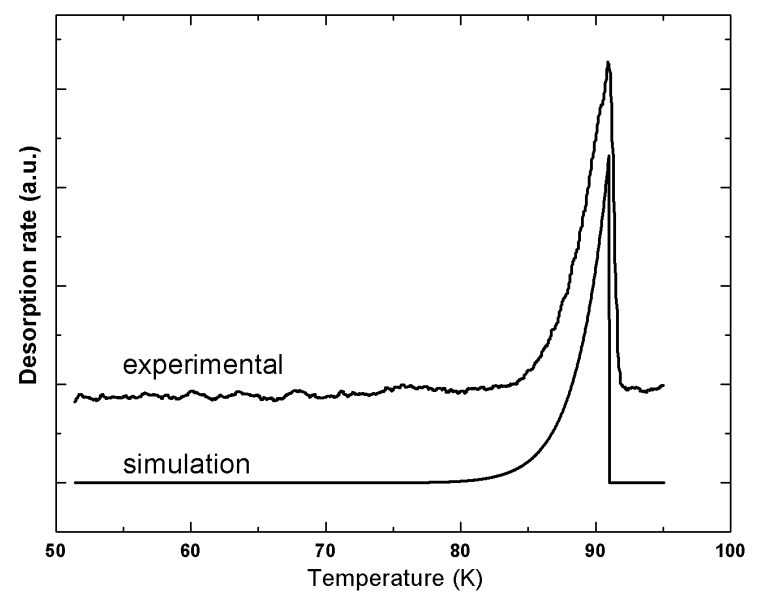

Fig. 5. $\mathrm{CO}_{2}$ zeroth-order desorption. experimental (upper signal) and theoretical (bottom signal), based on Equation 5. For clarity reasons we have shifted up experimental signal.

coefficient of 0.99991 and a slope of $\sim 42 \mathrm{~Hz} \mathrm{~K}^{-1}$ characterizes our system. These data are obtained in the range of temperatures between $95 \mathrm{~K}$ and the accretion process onset at $42 \mathrm{~K}$ (dotted arrow 1). Frequency during the experiment with and without
Table 2

$\mathrm{CO}_{2}$ sublimation energy compared with other results reported by NIST.

\begin{tabular}{|l|c|c|c|}
\hline & This work & NIST [18] & NIST [17] \\
\hline $\mathrm{T}_{\text {sub }}(\mathrm{K})$ & 92 & $139-195$ & $198-216$ \\
\hline $\mathrm{E}_{\text {sub }}\left(\mathrm{kJ} \mathrm{mol}^{-1}\right)$ & 27.6 & 26.3 & 26.1 \\
\hline
\end{tabular}

the influence of the temperature drift is plotted in Fig. 4 (top and bottom panel, respectively).

The derivative against temperature of the quartz signal variation, without the temperature influence, provides the desorption rate as a function of temperature (Fig. 5, upper signal). As can be observed in this figure, a sharp, asymmetric peak is located around $92 \mathrm{~K}$ (which is related to $\mathrm{CO}_{2}$ highest sublimation rate), an exponential increase of frequency is followed by a sudden drop to zero. This behaviour characterizes a zeroth-order kinetics. Fig. 5 (bottom signal) plots the synthetic curve obtained by using the Polanyi-Wigner equation (Equation 5) with kinetic parameters of $\mathrm{CO}_{2}$.

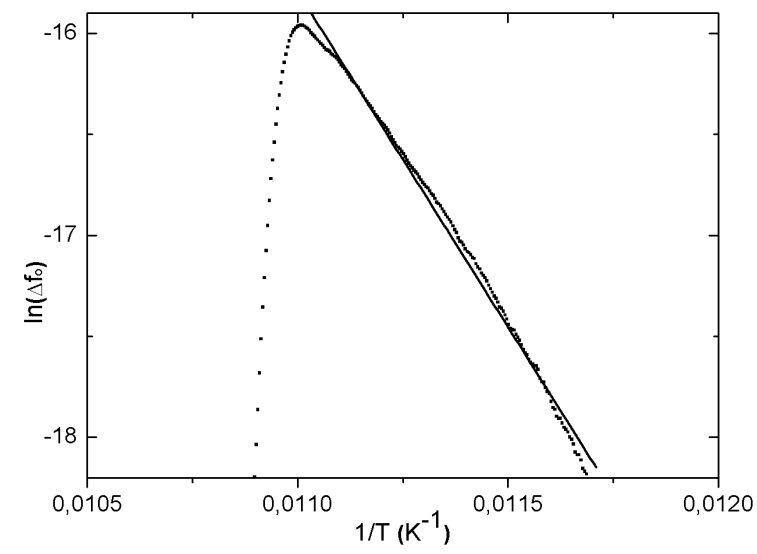

Fig. 6. The $\ln (\Delta f o)$ versus $1 / \mathrm{T}$ (dotted line) and linear fit (straight line).

The sublimation energy is obtained by a straight line fit of the plot $\ln \left(\Delta f_{0}\right)$ versus $1 / \mathrm{T}$, as displayed in Fig. 6. The regression coefficient obtained (0.993) allows us to assume that the preexponential factor and the activation energy are not temperature dependant, in the temperature range of desorption (80$92 \mathrm{~K}$, in this case).

\section{Results and discussion}

In this paper we show a new procedure developed to obtain the energy of sublimation of ices at low temperatures by using only one QCMB. 
Sublimation in a thickness controlled experiment under $\mathrm{HV}$ proceeds with zeroth-order kinetics (see Fig. 5). Plotting $\ln \left(\Delta f_{0}\right)$ versus $1 / \mathrm{T}$ a linear behaviour is shown along the leading edge of the QCMB signal during sublimation. This fact confirms the reliability of the method and setup presented in this paper.

There were no previous results of sublimation energy at this low temperatures for pure $\mathrm{CO}_{2}$. In table 2 this result is compared with other similar data (although obtained under different conditions) reported so far. From the table can be appreciated that a decreasing value in sublimation energy occurs as the temperature of sublimation increases. Slight differences can be explained because at temperatures close to $90 \mathrm{~K}$ a phase change (amorphous to crystalline) can be involved [19-21].

\section{Conclusions}

The energy of sublimation of molecules is a relevant parameter in several aspects of dynamic physics involving interactions between condensed and not condensed phases. In this paper we have presented a new method implemented to determine the sublimation energy.

This method presents several advantages respect to the previously used: The ability to work with other analysis techniques simultaneously (interferometry, Infrared and/or UV-visible spectroscopy, etc.), not possible when a Knudsen cell is used; simplicity respect other techniques present in the literature, because it is based in only one QCMB frequency variation; possibility to work under a wide range of vacuum conditions; and quickness in the procedure since it is not necessary to stop and restart the warming up.

\section{Acknowledgements}

This work was supported by the Ministerio de Educación y Ciencia (Co-financed by FEDER funds) AYA 2004-05382 and AYA 2007-65899.

\section{References}

[1] Zemansky M. W. McGraw-Hill book co., New York 1957

[2] Baierlein R. Cambridge University Press, Cambridge 1999
[3] Santos L. M. N. B. F., Schröder B., Fernández O. P., Ribeiro da Silva M. A. V., Thermochim. Acta, 415, 15-20, 2004

[4] Reichl, A Modern Course in Statistical Physics, John Wiley and Sons Inc. 2nd Revised edition, 1998

[5] Knudsen, Ann. d. Phys., 28, 75, 1909

[6] Torres L. A., Hernández-Contreras I., Guardado J. A., González M. G., Meas. Sci. Technol., 5, 51-54, 1994

[7] de Barros A. L. F., Medina A., Zappa F., Pereira J. M., Bessa E., Martins M. H. P., Coelho L. F. S., Wolff W., de Castro-Faria N. V., Nucl. Instrum. Meth. A, 560, 219-223, 2006

[8] de Kruif C. G., van Ginkel C. H. D., J. Phys. E, 8, 764766,1973

[9] Stefanov A., Stibor A., Domínguez-Clarimon A., Arndt M., J. Chem. Phys., 121, 6935-6940, 2004

[10] Bryson III C. E., Cazcarra V., Levenson L. L., J. Chem. Eng. Data, 19, 107-110, 1974

[11] Sack N. J., Baragiola R. A., Phys. Rev. B, 48, 99739978, 1993

[12] Teolis B. D., Famá M., Baragiola R. A., J. Chem. Phys., 127, 074507, 1-9, 2007

[13] M. Polanyi, E. Wigner, Z. Phys. 33, 429-434, 1925

[14] Luna R., Millán C., Santonja C., Satorre M. Á., Vacuum, 83, 942-948, 2009

[15] Sauerbrey G., Z. Physik, 155, 206-222, 1959

[16] Roux J. A., Wood B. E., Smith A. M., Plyer R. R., 1979, Report N AEDC-TR-78-81, Defense Technical Information Center

[17] Stephen R. M., Malanowski S., Handbook of the Thermodynamics of Organic compounds, Elsevier, New York, 1987

[18] Jones, A. H., J. Chem. Eng. Data, 5, 196-200, 1960

[19] Falk M., J. Chem. Phys., 88, 560-564, 1987

[20] Sandford S. A., Allamandola L. J., The Astrophysical Journal, 355, 357-372, 1990

[21] Luna R., Millán C., Domingo M., Satorre M. Á., Astrophys. Space Sci., 314, 113-119, 2008 